\title{
Características lingüísticas de los textos de ciencias sociales en la formación del profesorado
}

Recibido: mayo 2012

\author{
Teodoro ÁLVAREZ ANGULO \\ talang@edu.ucm.es \\ Javier ÁLVAREZ REGIDOR \\ javier.alvarez.regidor@gmail.com \\ Grupo Didactext \\ (http://www.didactext.net)
}

Aceptado: enero 2013

\section{RESUMEN}

Este artículo plantea la necesidad de conocer las peculiaridades lingüísticas y textuales de los textos de ciencias sociales relacionados con la formación del profesorado. Pretende hacer consciente a estudiantes y a profesores de la importancia que tiene conocer la "gramática de la exposición" de textos de Ciencias Sociales, de manera que comprendan mejor y se expresen con precisión cuando escriban estos textos.

Palabras clave: textos de ciencias sociales, características lingüísticas y textuales, conciencia del proceso de escritura, competencia escrita.

Caractéristiques linguistiques des textes de sciences sociales dans la formation des professeurs

\section{RÉSUMÉ}

Cet article insiste sur le besoin, pour les étudiants et les professeurs, de connaître les singularités linguistiques et textuelles des textes de Sciences Sociales en rapport avec la formation des professeurs. Nous voulons que les étudiants et les professeurs deviennent conscients du fait que maîtriser la " grammaire de l'exposition » des textes des Sciences Sociales est un atout pour mieux comprendre et mieux s'exprimer à l'heure d'écrire ce type de textes.

Mots-clé: textes de sciences sociales, caractéristiques linguistiques et textuelles, conscience du processus d'écriture, compétence écrite.

\section{Linguistic characteristics of the social science texts related with the teacher training}

\begin{abstract}
This article raises the need of knowing textual and linguistic characteristics of the social science texts related with the teacher training. The aim has been to assess the importance of knowing "the grammar of the exposition" of social science texts to teachers and students in order to get a better understanding and expression when they write these types of texts.
\end{abstract}


Keywords: social science texts, textual and linguistic characteristics, conscience of the writing process, and writing competence.

SUMARIO 1. Presentación. 2. ¿Qué es un texto expositivo? 3. Gramática de la exposición de información. 3.1. En el nivel textual. 3.2. En el nivel oracional. 3.3. En el nivel léxico. 3.4. En el nivel morfológico. 4. Conclusiones. Referencias bibliográficas

\section{PRESENTACIÓN}

Uno de los principios en que se asienta la competencia en comunicación lingüística de los individuos es su capacidad para comprender y producir textos en diferentes situaciones, con intenciones distintas, lo que configura géneros discursivos asimismo diferentes (ADAM, 1992).

Leer y escribir, en ámbitos académicos, son habilidades o competencias, a la vez que herramientas de aprendizaje para adquirir información y construir conocimiento (BEREITER Y SCARDAMALIA, 1987). Comprender y redactar un texto consiste en pensar de forma más o menos simultánea y permanente, además del contenido informativo del mismo, en cuestiones como: a quién va dirigido (destinatario, audiencia); qué se pretende conseguir con él (intención); cómo está organizado o cómo se ha de organizar (fases del proceso); cómo se ha de transmitir (género discursivo, normas de textualidad y regularidades lingüístico-textuales); y cómo motivar y tutelar el proceso de escritura, hasta llegar al texto final. De ahí la importancia de conocer (y enseñar) los géneros académicos que se usan para construir conocimiento científico en cada materia del saber, de manera que se pueda proporcionar a los estudiantes los conocimientos y las herramientas necesarios para comprender y expresarse en contextos científicos (presentación, estructura y organización de la información) (TEBEROSKY, 2007).

Esta relación permanente entre destinatario e intenciones; género, normas de textualidad y peculiaridades lingüísticas; y el uso real de la escritura mediante intereses de los alumnos traducidos en proyectos de escritura (CAMPS, 2003) favorecen la adquisición de información (saber enciclopédico), a la vez que el aprendizaje del proceso y del género correspondiente (función epistémica de la escritura). En esto reside la complejidad del aprendizaje y la enseñanza de esta habilidad lingüística.

Respecto del propósito de exponer información, se puede afirmar que un discurso o texto "expositivo", "explicativo" o "académico" es aquel que tiene como finalidad transmitir, exponer y explicar información, por parte de quien conoce un tema, a quien o a quienes quieren saber sobre él. Estos textos, como sucede con los demás tipos, tienen una serie de características o regularidades, tanto en su configuración mental o esquemática, como lingüísticas y textuales. En consecuencia, se puede afirmar que tienen "su propia gramática" (ÁLVAREZ, 2001, 2005 y 2010). 
Conviene resaltar que los estudios sobre el uso de la lengua en contextos académicos, distinguen entre la lengua de instrucción, cometido de todo profesor en cada materia (cuestión que destacan de modo particular movimientos como Wtiting Across the Curriculum y Writing in the Disciplines) y la lengua como disciplina, que estudia el sistema de la lengua y la actividad metalingüística que requiere leer y escribir, y que es competencia principal de la asignatura de Lengua Castellana y Literatura (BAZERMAN et al., 2005). Esto, por otra parte, evidencia una concepción epistémica de la escritura, en cuanto que se defiende que se adquiere a la vez información sobre el tema y sobre las peculiaridades del género discursivo que lo vehiculará.

Este trabajo se orienta a la formación académica y profesional de maestros y de profesores, tanto en la formación inicial como permanente. Con el fin de contribuir a un mayor conocimiento de las peculiaridades lingüísticas y textuales de los textos del ámbito de las Ciencias Sociales, en contextos académicos y escolares, analizamos un corpus de textos extraídos de sendos manuales de Geografía [G], Historia $[\mathrm{H}]$, Historia del Arte [HA], y Didáctica de las Ciencias Sociales [DCCSS], recomendados por profesores del Departamento de Didáctica de las Ciencias Sociales de la Universidad Complutense de Madrid. Subrayamos, en cada caso, el o los fenómenos contenidos en cada muestra. Los datos que nos proporciona el análisis de este corpus nos permite establecer una serie de características, que es necesario conocer para facilitar la comprensión y la producción de textos académicos en este campo del conocimiento.

\section{2. ¿QUÉ ES UN TEXTO EXPOSITIVO?}

Los textos expositivos se caracterizan por una voluntad de hacer comprender -y no solamente decir- determinados fenómenos. Buscan modificar un estado de conocimiento; por ello, de manera más o menos explícita, suele aparecer una pregunta como punto de partida, que, a lo largo del texto, se ha de ir resolviendo. Se trata con estos textos de transmitir datos, organizados con objetividad y rigor. Es común concebir estos textos como aquellos cuyo objetivo principal es expresar información o ideas con la intención de mostrar y de explicar o hacer más comprensible dichas informaciones. Por tanto, son los textos más frecuentes en el ámbito académico y escolar, y, sin embargo, no es habitual que el profesorado enseñe a leer y a escribir textos de especialidad, en las clases de historia, de geografía, de ciencias, de matemáticas, etc.

Todo ello sucede a pesar de las abundantes referencias que se hace a la lectura y a la escritura en los currículos de las diferentes áreas de conocimiento, así como de los comentarios insistentes de los profesores, en este sentido. Algunas de estas referencias son: el estudio de propiedades, características y componentes de una determinada estructura (descripción); la argumentación mediante razonamientos lógicos; la recogida, selección y procesamiento de la información básica, a partir 
de fuentes diversas; la descripción de acontecimientos históricos y geográficos; la adquisición y utilización correcta y precisa del vocabulario específico; etc. Por ello, WRAY y LEWIS (1997/2000) abogan por la necesidad de introducir desde los inicios de la escolaridad la enseñanza de los textos de información y la forma de aprender de ellos. Suelen ser los textos narrativos los que merecen mayor atención, desde los primeros años de la educación.

La exposición de información obedece a un esquema prototípico que está formado por tres elementos: problema - resolución - conclusión.

Cuadro 1. Esquema prototípico de la exposición de información.

(Tomado de J.-M. Adam (1992)

Fase de pregunta + Fase resolutiva + Fase de conclusión

(Problema) (Resolución) (Conclusión-

¿Por qué? (Porque) Evaluación)

¿Cómo?

Ahora bien, estos textos no responden a una superestructura común, sino que se ajustan a distintas maneras básicas (subtipos) de organizar la información, como son: descripción y definición, clasificación-tipología, comparación y contraste (semejanzas y diferencias), problema-solución (pregunta-respuesta), causaconsecuencia (Más información en ÁLVAREZ, 2001).

Pertenecen a la exposición los siguientes géneros discursivos: a) discursos científicos: artículos, monografías, tratados, exposiciones y cursos; b) discursos de divulgación: artículos de revista, conferencias, enciclopedias y documentos; c) discursos didácticos: manuales y documentación oficial. Su función consiste en transmitir información. Se trata de textos que tienen la pretensión de exponer o explicar información o conocimientos en los diferentes campos del saber y su aplicación, de divulgar información. Y estos son los textos con los que se enfrenta diariamente el alumno, tanto en la lectura (libros de texto, materiales didácticos y documentación en general), como en la escritura (trabajos y exposiciones, dosieres o portafolios, exámenes). Comparados con los textos narrativos, los textos expositivos se diferencian principalmente por lo siguiente: a) requieren una lectura más atenta; b) permiten hacer menos predicciones; y c) suscitan menos inferencias (SÁNCHEZ MIGUEL, 1993).

Esto pone de manifiesto la paradoja que supone el hecho de que sea este el tipo de texto más frecuente en la vida académica y, sin embargo, no se corresponda con la necesaria ejercitación sistemática en las áreas del currículo; por tanto, es muy común que la exposición sea el tipo de texto que más utilizan los estudiantes y el que menos dominan. 


\section{GRAMÁTICA DE LA EXPOSICIÓN DE INFORMACIÓN}

A continuación mencionamos las principales características lingüísticas y textuales que aparecen regularmente en estos textos, distribuidas en distintos niveles lingüísticos y textuales (ÁLVAREZ, 2001 y 2010). Aportamos, en cada caso, muestras extraídas del corpus de textos estudiados, con el fin de ilustrar dichos fenómenos. Son las siguientes:

\subsection{En el nivel textual}

- Recursos tipográficos. Su función textual consiste en favorecer la organización interna y las relaciones intertextuales. Son los siguientes: Tipo de letra, guiones, números o letras para enumerar hechos o elementos, control de márgenes, comillas, subrayados y uso de paréntesis.

Son muy frecuentes los recursos tipográficos para destacar términos y conceptos clave o núcleos temáticos, expresados en negrilla; expresiones extranjeras, en cursiva; comillas y cursiva para referencias bibliográficas; uso de paréntesis explicativos y de indicadores de autoría y de referencias.

- Formas supralingüísticas: Títulos, subtítulos, epígrafes. Contribuyen a organizar de forma lógica la información en el texto.

De una manera u otra, en función de las normas de redacción exigidas por el editor, todos los textos manejados diferencian los títulos de capitulo, los subtitulos, los epígrafes y subepigrafes, mediante la oposición mayúsculas, minúsculas; negrilla, cursiva; o mediante el tamaño de la letra, además de la numeración correspondiente, que consta en el texto y en el índice.

\section{- Citas, referencias y autoría}

Se trata de procedimientos textuales para fundamentar en fuentes el contenido de lo que se pretende tratar en el texto. Las citas son en algunos casos literales, en otros se trata de glosar ideas, temas o datos contenidos en la obra de referencia. Cada procedimiento tiene su representación formal, conforme a las normas de redacción que establezca el editor. Algunos ejemplos de ello son:

[DCCSS1] La generalización de la enseñanza de la Geografía en el siglo XIX

Así, como ya señalaron Capel y Urteaga (1986), "ha estado presente con continuidad, y casi siempre con autonomía, en la enseñanza primaria de todos los países europeos". 
[DCCSS2] La orientación de los contenidos condiciona la didáctica de la materia.

Un caso destacado de lo que decimos fue el Grupo Germanía 75 (Prats y Paniagua, 1977), nacido en Valencia, que, asumiendo las nuevas corrientes historiográficas cercanas al marxismo, planteó, con desigual éxito (Prats, 1989), la enseñanza de la Historia con un método que intentaba elaborar un discurso histórico con la construcción de los conceptos y las teorías propias de la orientación historiográfica que defendía.

[G1] Los afluentes

La cuenca del Ebro (Davy, L., 1978) recibe derrames pirenaicos, cantábricos e ibéricos.

[G1] Análisis hidrológico del caudal

Según las estimaciones del Plan Hidrológico Nacional (fig. 4.4), las pérdidas de agua precipitada y no desaguada en el mar sobrepasan el $80 \%$ en los ámbitos de las Confederaciones Hidrográficas del Guadiana, Guadalquivir, sur de España, Segura y Júcar.

[G2] Características de la precipitación y tipos de precipitación

Como ha expresado en varias ocasiones la OMM [Organización Mundial de Meteorología], el problema de la supresión del granizo ha llegado a un punto en el que cualquier otro progreso significativo en materia de aplicaciones deberá esperar a que se resuelvan varios problemas cientificos fundamentales.

[H1] La década de la radio

La prensa deportiva, con un discurso bien trabado, animaba a la socialización de todos los valores de nacionalismo exaltado con tono castizo y grandilocuente. $Y$ buena muestra de ello fue el diario Marca, publicación del Movimiento, que alcanzó una tirada de 200.000 ejemplares.

[H1] La religión Católica: ritos colectivos y moral social

En el Jarama de Sánchez Ferlosio, publicada en 1955, quedaron ejemplarmente retratados en una excursión los códigos, actitudes y conversaciones de la juventud de la época.

[H2] Historia atlántica. Un debate historiográfico en Estados Unidos

Dos obras publicadas en el año 2009 nos servirán para enmarcar nuestro análisis. Por un lado, el libro editado por Bernard Bailyn y Patricia L. Denault, Soundings in Atlantic History: Latent Structures and Intellectual Currents, 15001830, y el publicado por Jack P. Greene y Philip D. Morgan, Atlantic History: A Critical Appraisal. 
[HA1] Las artes durante la Edad de Bronce Antiguo en Europa

La documentación arqueológica sobre este periodo, en la dilatada extensión geográfica de Europa, desde el Cáucaso hasta las islas Británicas, es abrumado-

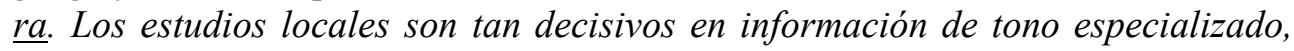
que es difícil dibujar una panorámica a grandes rasgos.

[HA1] Wessex, la Armórica y Mecenas

En 1938, Stuart Piggott presenta a la Prehistoria europea una contribución excepcionalmente brillante: la cultura de Wessex.

\section{- Descripciones, definiciones o conceptualizaciones}

Mientras que la descripción literaria no siempre es vista con buenos ojos (La descripción me da mucha pereza y creo que los lectores se la suelen saltar en el noventa por ciento de los casos, a no ser en novelas como las de Juan Benet, que son maravillosas porque él era un topógrafo que escribía, afirma Bryce Echenique; o, como dice Umbral, en Mortal y rosa, el arte descriptivo, minucioso, es pueril y pesado. El arte expresivo, expresionista, aísla rasgos y gana, no sólo en economía, sino en eficacia, porque arte es reducir las cosas a uno sólo de sus rasgos, enriquecer el universo empobreciéndolo, quitarle precisión para otorgarle sugerencia.

No sucede lo mismo con los usos de la descripción en la escritura expositiva, en cuanto que está relacionada con conceptos tales como: estructura, morfología, procedimiento, proceso, mecanismo, sistema, comparación, funciones, fases, ciclos, etc. La descripción proporciona conocimientos de un tema, de un objeto o de un fenómeno por los accidentes, las partes, las características, las propiedades, etc., que le son propios y que lo determinan con relación a otros. Describir es realizar un tránsito desde la observación al análisis (discriminación de partes, componentes, aspectos...). Para conseguir describir con éxito un determinado fenómeno es preciso tomar notas que favorezcan la sistematización (el orden) de las ideas, como puede verse en la figura 1.

De hecho, la descripción se considera como un texto museo, en cuanto que pone de manifiesto la competencia enciclopédica, la competencia léxica, la competencia taxonómica y la competencia axiológica del individuo.

Con el fin de facilitar la ejercitación de la descripción, es conveniente ayudarse de guías de ayuda basadas en cuestionarios que medien y dirijan la observación e inicien en la descripción de personas, paisajes, objetos, etc. (ÁLVAREZ, 2010).

La definición, a su vez, se relaciona con ideas tales como: concepto, ley, teorema, axioma, principio, etc. Implica delimitación de una categoría con respecto a otras. 
La consideración de ambos subtipos de organización mental de la exposición (particularizante, la descripción; y generalizadora, la definición) supone un recurso importante para la mejora de la comprensión y la producción de textos en todas las materias del currículo y en los usos orales y escritos de toda interacción social.

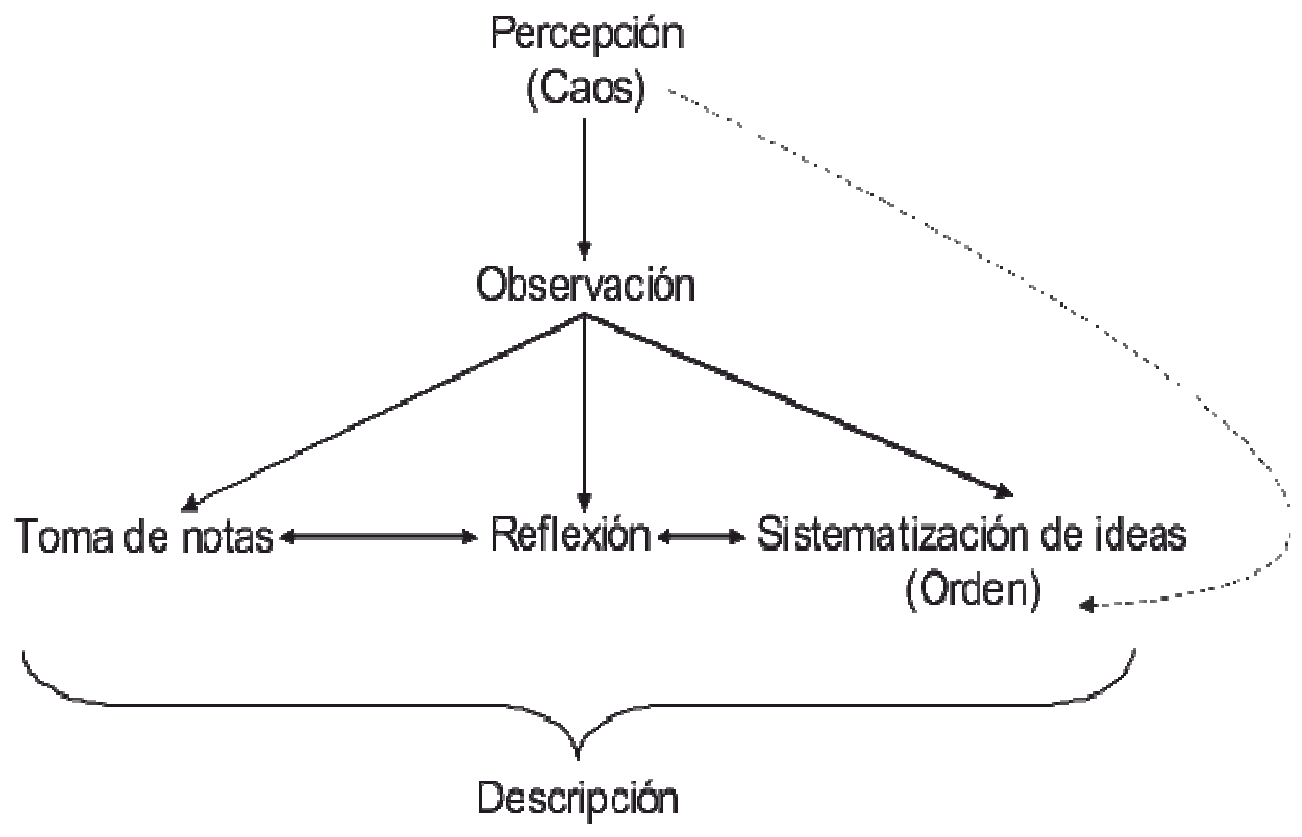

Fig. 1. Complejidad del proceso de descripción.

(Del caos de la percepción al orden del texto y del discurso)

La figura muestra el recorrido desde la percepción (global, caótica) hasta la sistematización de ideas (el orden) que exigen la descripción y la definición, tanto en la comprensión como en la producción de textos.

Las funciones de definición y de descripción tienen una presencia muy notoria en estos textos, habida cuenta de que pretenden explicar la información que se expone, de manera que la entienda bien el lector. La manera de exponer la descripción, con frecuencia recurre a las características, la clasificación, etc., con el fin de mostrar las partes de un todo, de manera que se pueda entender mejor. En los textos trabajados, los más abundantes en definiciones y descripciones son los de Geografía y de Historia del Arte. . 
Veamos algunos ejemplos.

[DCCSS2] La orientación de los contenidos condiciona la didáctica de la materia

En relación con estos cambios, puede afirmarse que en la mayor parte de Europa, la tendencia general, aunque no coincidente en todos los paises, ha sido la de contemplar la historia como una ciencia social que sirva para educar la conciencia colectiva de los ciudadanos así como para reconocer e identificar las raices sociales, políticas y culturales de las diferentes naciones, intentando evitar su manipulación del conocimiento del pasado y excluyendo el fomento de posiciones xenófobas (Caballi, 2005).

[DCCSS3] La selección y secuenciación de los contenidos sociales

El concepto de transposición didáctica designa el paso del conocimiento social científico o "saber sabio" al saber enseñado (Chevallard, 1991) y reconoce la obligada distancia que los separa.

[DCCSS3] Los contenidos conceptuales. La selección de los conceptos disciplinares

Podemos decir que saber Historia, Geografia, Arte o Economía, etc. es, ante todo, poseer redes o sistemas jerarquizados de conceptos que sirven para tratar los problemas de esta materia. Enseñar Geografía, por ejemplo, es ayudar a construir un cuerpo de conceptos que son transversales a toda la Geografía, como los conceptos de extensión, distancia, escala, localización, distribución, centralización, accesibilidad, doblamientos, territorio, etc.

[G2] 4. Características de la precipitación

El epigrafe 4 se desarrolla mediante los siguientes subepigrafes: 4.1. Cantidad; 4.2. Intensidad, duración y frecuencia; 4.3. Variabilidad

El desarrollo de los diferentes subepígrafes representa por sí mismo la explicación del concepto "precipitación", mediante lo característico que se pretende explicar.

[G2]5. Tipos de precipitación

Este epígrafe se desarrolla a través de los subepigrafes siguientes: 1 . precipitación ciclónica y frontal; 2. Precipitación por convergencia; 3. Precipitación convectiva.

Los distintos subepígrafes describen y definen los tipos de precipitación. 
[HA3] La arquitectura del siglo XVIII en Potosí

La portada (fig. 500), con sus columnas dispuestas en dos planos, presenta una planta un tanto movida, y el desplazamiento del segundo cuerpo hacia arriba permite disponer un frontón curvo con una hornacina y roleos en el tímpano. Las columnas salomónicas de cinco espiras están superpuestas a pilastras con estrías sesgadas, y tienen la fila de hojas en el imoscapo, que se repite sobre la anilla que las ciñe a la altura del primer tercio. Un grueso tallo se enrosca por la parte gruesa de las columnas, volcando sobre ella racimos y hojas, y un motivo imbricado llena las concavidades del fuste retorcido. Los netos de los pedestales y los recuadros de las jambas y de la rosca del arco están decorados con cuadrifolias. Frontones curvos, rellenos con veneras, como los que veremos, pero algo más estilizados, en San Benito o San Bernardo, rematan los laterales del primer cuerpo; hojas muy estilizadas animan el friso, y, para no dejar espacio libre entre el arco y su encuadramiento, unas grandes hojas acarician el trasdós mientras los peciolos enrollados en espiral, convergen hacia el ángulo de la enjuta.

\section{- Ilustraciones}

Son muy abundantes las ilustraciones en todos los textos manejados; en menor medida aparecen en los de Didáctica de las Ciencias Sociales. Adoptan formas diferentes, tales como: cuadros, figuras, esquemas, gráficas, hidrogramas, mapas, vistas panorámicas, climogramas, perfiles longitudinales, tablas comparativas, fotografias, láminas, mapas conceptuales, ejes cronológicos, pirámides de población, documentos y textos, etc. Desde un punto de vista pragmático, aparece tras indicaciones del tipo: Como se puede ver en el gráfico...; Observa la(s) fotografia(s) de la página...; En este dibujo...; Observa los diagramas...; y otras semejantes.

\section{- Enumeraciones}

La enumeración se concibe como el grado cero de la descripción, en cuanto que, de forma paratáctica, relaciona los componentes de un todo, los aspectos de un fenómeno, etc., lo que hace de este fenómeno lingüístico en los usos de la lengua un recurso importante de descripción. Veamos algunos ejemplos:

[G1] Lagos y zonas húmedas

Gran número de vocablos (lago, laguna, estanque, estaño, marisma, tollo, tremedal, salma, tabla, estero, albufera, nava, marjal, galacho, ibón, charca, etc.) registran la variedad de zonas húmedas de España/Tello, T., y López Bermúdez, F., 1988).

[HA1] Esplendor y exotismo en Europa occidental

La Prehistoria europea vuelve a sorprender -y ésta no será la última vez- por el afán de lujo que transmiten una amplia serie de objetos arqueológicos. Éstos 
pertenecen a la categoría de los adornos personales (pendientes, brazaletes, collares, complementos del vestido, etc.), de las enseñas de poder (hachas ceremoniales, mazas de mando, armas de prestigio, etc.), o de los enseres de distinción (vajillas de oro, de concha o de ámbar).

[HA1] Stonehenge y la cultura del vaso campaniforme

[Stonehenge] Son arqueros y guerreros. Sus tumbas individuales contienen placas rectangulares de piedra perforadas, que sirvieron de protección de la muñeca en el uso del arco; puntas de flecha de sílex; hojas de cuchillos y dagas de metal (de cobre, fundamentalmente); hachas de piedra de batalla; y algún objeto pequeño de auténtico bronce.

\section{- Comparaciones}

La comparación es un fenómeno textual recurrente en los textos que pretenden transmitir información. Veamos unos ejemplos:

[G1] Los volúmenes de escorrentía fluvial

A efectos comparativos, los recursos unitarios del sureste peninsular son quince veces inferiores al norte de España (fig. 4.5).

[HA1] La Edad de Bronce

Pocas contribuciones a la historia de la Humanidad han tenido tanta trascendencia en el dominio de la naturaleza como la adquisición de los medios materiales y técnicos para producir objetos de bronce.

[HA1] Las artes durante la Edad de Bronce Antiguo de Europa

En la fase cultural del Bronce Antiguo, el continente europeo se coloca a la altura de las grandes civilizaciones de la Antigüedad.

[HA2] Conquistadores del mundo

Sea como fuese, conocemos a Pompeyo, Augusto y Nerón o Tito, casi como si hubiéramos visto sus rostros en los noticiarios.

[HA3] La arquitectura del siglo XVIII en Potosí

Dificil será encontrar un modelo de campanario comparable a la espadaña de la Compañia, cuya silueta se recorta sobre el limpio cielo de Potosí con la grandiosidad y el empaque de un arco de triunfo de la Roma antigua (fig. 501).

\section{- Ejemplificaciones}

Es frecuente el recurso a la ejemplificación de fenómenos que se explican en el texto. Algunos casos son: 
[DCCSS2] Temas que plantean las ideas de cambio y continuidad en el devenir histórico

Incluso en acontecimientos tan paradigmáticos como la Revolución francesa, por citar un caso, podemos encontrar motivaciones y elementos que son un claro fruto de un rebrote de elementos tradicionales que, sin embargo, impulsan la propia revolución. Debido a ello, es preciso incorporar elementos históricos que consideren y ejemplifiquen la pervivencia del pasado en sociedades que, en apariencia, ya han roto con él.

[DCCSS3] La selección y secuenciación de los contenidos sociales

Veamos, a modo de ejemplo, nuestra propuesta para la Geografía en el Bachillerato.

[G1] Grandes ríos peninsulares

A modo de ejemplo, sólo se considerarán la indigencia del Segura y la abundancia del Ebro.

\section{- Fórmula de cierre}

A pesar de ser los textos analizados capítulos de libros, resulta común que se cierre el capítulo mediante un párrafo resumidor o concluyente, del tipo de los que siguen.

[DCCSS1] Abstracción y conceptualización como finalidad

Todo ello constituye un repertorio de nociones geográficas imprescindibles para adquirir una correcta concepción del entorno en el que se desarrolla la vida y la actividad de cualquier individuo de la sociedad contemporánea.

[DCCSS2] Epígrafe denominado "Conclusión" en el cap. 2. Los contenidos en la enseñanza de la historia

[HA1]. La Edad de Bronce

Las conclusiones a extraer sobre la naturaleza del poder en la cultura otomana, basadas en las implicaciones sociales de sus poblados amurallados, son, por tanto, sólo parciales, aunque de aplicación general.

[HA2]. Conquistadores del mundo

Ello [los retratos de este periodo, siglos IV y V] nos revelan a la gente que presenció, y finalmente aceptó, el nacimiento de la cristiandad, que era tanto como la terminación del mundo antiguo. 
- Reformulaciones discursivas. Consiste este fenómeno lingüístico en decir lo mismo con otras palabras, más fáciles de entender, a juicio del emisor. Se trata, por tanto, de una paráfrasis, para lo cual se utilizan expresiones, tales como: o sea, es decir, esto es, en otras palabras, dicho de otra manera, asi sucede, etc.

Algunos ejemplos son:

[DCCSS3] La selección y secuenciación de los contenidos sociales

... es decir, se demuestra la necesidad de proceder a una transposición didáctica adecuada del conocimiento social científico.

[G1] Componentes del caudal fluvial

En efecto, a lo largo de este tramo [subsistema acuifero de Albacete] y sin ningún afluente significativo, el Júcar aumenta su caudal en unos 300-450 hm3 (esto es, entre $10-15 \mathrm{~m} 3 / \mathrm{s})$.

[G1] Componentes del caudal fluvial

La escorrentía superficial comprende el flujo superficial del aguan en dirección a los cauces. Incrementa el caudal de los ríos desde el momento que se rebasa la capacidad de infiltración (fig. 4.8). En otras palabras, la escorrentía superficial depende de la estructura espacio-temporal de la precipitación y de la saturación del suelo.

[G1] Grandes ríos peninsulares

El sector de la cabecera (unos $5.000 \mathrm{~km} 2$ ) proporciona $631 \mathrm{hm} 3$ interanuales, esto es, el 65\% de los recursos hídricos del Segura.

[H2] Historia atlántica. Un debate historiográfico en Estados Unidos

Es decir, además de ensalzar las conexiones y las relaciones de los flujos atlánticos, propone profundizar en las diferencias y similitudes entre las distintas sociedades coloniales.

\section{- Otros procedimientos de explicación}

Los recursos de proceso y de estructura, mecanismos descriptivos por excelencia, se expresan de formas diversas, a través de las fases, los pasos, las etapas, los estadios, los niveles, las partes, los componentes, etc., elementos que forman el todo que configura el proceso o la estructura. Estos términos o expresiones sirven de elemento introductor de la función descriptiva que desempeñan.

Veamos un ejemplo. 
[HA1] El carro solar de Trundholm

El procedimiento de la cera perdida en hueco se llevó a un mayor grado de maestría en la realización del caballo. Sigamos los pasos...

\subsection{En el nivel oracional}

En la construcción sintáctica del texto expositivo son frecuentes las oraciones simples y las oraciones compuestas coordinadas, yuxtapuestas y subordinadas sustantivas, adjetivas y adverbiales (causales, consecutivas, comparativas, concesivas y temporales). Es importante destacar que la exposición de información muestra preferencia por las construcciones lógicas sobre las psicológicas, establecidas sobre el esquema sintáctico de sujeto-verbo-complementos.

- Aposiciones explicativas, uso de los dos puntos introductorios, que explicativo, inciso explicativo, paréntesis explicativos. Estos fenómenos textuales tienen la función pragmática de introducir en el texto información nueva; de ahí la importancia de conocer estas características lingüístico-textuales en la exposición de información. Ejemplo de esto son las siguientes muestras textuales:

[DCCSS3] La selección y secuenciación de los contenidos sociales

Esta disociación de conceptos o desintetización del modelo científico que luego debe ser recompuesto progresivamente, parece inherente al proyecto didáctico.

[G1] Las aguas

El agua -uno de los cuatro elementos de las cosmografías clásicas- ocupa un lugar central en la organización de los ecosistemas naturales y en la morfología de los paisajes culturales.

[G1] Las aguas

Las terrazas -antiguos llanos de inundación abandonados por encajamiento fluvial-constituyen el rasgo geomorfológico habitual de las márgenes de los ríos.

[G3] La emergencia de los estados-nación europeos

El cinturón central europeo - básicamente las actuales Alemania y Europacontinuó siendo un rompecabezas de minúsculas unidades territoriales.

[G3] Bélgica

Los valones (42\%), que pueblan el sur de Bélgica, hablan y escriben francés.

[H1] La Edad de Bronce

Los metalúrgicos (operadores del cobre, del bronce o del oro) son gentes extraordinariamente capacitadas para su oficio. 
[H1] La Edad de Bronce

El broncista pronto hubo de aprender que la inclusión de la casiterita (el óxido, la piedra del estaño) producía mejores resultados que el arsénico en la elaboración y en el acabado de los objetos de bronce.

[H1] La religión católica: ritos colectivos y moral social

Con la afluencia de turistas, convertida pronto en el maná externo del régimen, que se disparó en 1951-700.000 visitantes, aproximadamente- para duplicarse a mediados de la época -un millón y medio de turistas-, llegaron ideas y costumbres que chocaron vivamente con el despliegue del pudor colectivo y de la moral católica.

[H1] Los cambios de la sociedad campesina y el modo de vida urbano. Los primeros síntomas de la modernización

Las tiendas comenzaron a poblarse con más productos, aunque todavía existían dificultades para obtener algunos, como los yogures o el jamón York, asociados todavía durante muchos años a comida especial para enfermos.

[H1] La cultura crítica

Muchos, hijos del régimen, vástagos de las clases acomodadas, estaban ligados a las aulas universitarias.

[H2] Historia atlántica. Un debate historiográfico en Estados Unidos

Los dos grandes aliados durante la guerra, Estados Unidos y Gran Bretaña, ya habian enarbolado un pasado común para atraer a la hasta entonces reticente opinión pública estadounidense hacia la decisión de entrar en la contienda.

[HA1] La Edad de Bronce

El ingrediente del arsénico, natural o artificialmente añadido, ocasiona en la fusión la emanación de gases perniciosos, con el consiguiente peligro para la salud del broncista.

[HA1] La Edad de Bronce

La adquisición de ámbar se limita a su exclusivo lugar de origen en Europa: las orillas del mar Báltico (las costas de Letonia, de Lituania y la occidental de la península danesa de Jutlandia).

[HA2] Conquistadores del mundo

Hemos visto que Pompeya, una ciudad romana,... 
- Uso endofórico de los deícticos. Se trata de marcas o indicadores que refieren a otros elementos del texto que pueden ir delante (anafóricos) o detrás (catafóricos); esta función hace que sean elementos importantes en la cohesión textual. Su función consiste en situar al lector en el texto ("llevarlo de la mano") para que comprenda mejor. Así, es frecuente que aparezcan elementos anafóricos: lo dicho anteriormente; como ya se dijo; etc. Y catafóricos: más adelante diremos; a continuación señalamos; etc. Veamos algunos ejemplos.

[DCCSS3] La selección y secuenciación de los contenidos sociales

Hasta ahora se entendía por contenidos los hechos, los conceptos y los principios disciplinares, pero los nuevos currículos para la enseñanza obligatoria en España incorporan dentro de este capitulo los contenidos procedimentales y actitudinales.

[DCCSS3] La selección y secuenciación de los contenidos sociales

Diferenciamos en este capitulo los contenidos transdisciplinares o conceptos clave paradigmáticos y los conceptos disciplinares.

[G2] Características de la precipitación y tipos de precipitación

Varias de estas características son analizadas a continuación.

[HA2] Conquistadores del mundo

Hemos visto que Pompeya, una ciudad romana, contenía muchos reflejos del arte helenisticos...

[HA3] La arquitectura del siglo XVIII en Potosí

El sencillo esquema de esta portada [alusión a la figura 506] es semejante al que veremos en la de San Lorenzo, que se estaba labrando unos años más tarde, y la decoración ofrece temas que también hemos de encontrar en ejemplares de la misma época.

- Abundancia de conectores lógicos. Son elementos lingüísticos que enlazan las unidades del texto (apartados, párrafos, oraciones, etc.) ordenándolas y estableciendo una relación significativa entre ellas. Pueden pertenecer a varias categorías morfológicas: conjunciones (o, pero...); adverbios (seguramente, después...); locuciones (siempre que, no obstante...); sintagmas (a pesar de que...); e incluso oraciones (debemos aclarar que, hemos visto anteriormente que...). Los más frecuentes en estos textos son los conectores lógicos de adición, de continuación y de enumeración; de causa y consecuencia; de comparación, de ejemplificación; de explicación, aclaración y reformulación; de fuente, origen y autoría; marcador de conclusión o cierre; y resumidor. Omitimos la presentación de muestras, como se 
puede comprender, debido a la gran presencia de estos fenómenos lingüísticos. Remitimos a ÁLVAREZ (2005:45-47) para un mayor conocimiento de estas marcas o indicadores lingüísticos.

\subsection{En el nivel léxico}

En la exposición de información, el léxico tiene como características principales el monosemantismo y la denotación, lo que representa la búsqueda de la claridad, la precisión, la concisión y el rigor ("le mot juste", como dice Flaubert). O, como afirma A. Muñoz Molina: Escribir bien es pedirle a la inteligencia el nombre exacto de las cosas. Ya antes postulaba Lavoisier, en su Tratado elemental de Química (1789), que como son las palabras las que conservan y transmiten las ideas, resulta que no se puede perfeccionar la lengua sin perfeccionar la ciencia, ni la ciencia sin la lengua; y por muy ciertos que fuesen los hechos y muy justas las ideas que los originaron, sólo transmitiríamos impresiones falsas si no tuviesen expresiones exactas para denominarlas.

En la ciencia, la forma con que se reviste la verdad forma parte de la verdad misma. No es, pues, cualidad accesoria, sino esencial. La verdad es, por sí misma, por definición, clara, y el arte de la claridad es, por consiguiente, factor científico de primera categoría (Gregorio Marañón, La Medicina y nuestro tiempo, 1954). En consecuencia, postula N. Sanmartí (Aprendre ciències tot aprendre a escriure ciències. Barcelona: Ediciones 62, 2003) que el proceso de construcción del conocimiento científico comporta pasar de hablar un lenguaje personal, impreciso y con muchas expresiones importadas del conocimiento cotidiano, a ser capaces de utilizar el de la ciencia, mucho menos polisémico.

Por eso, probablemente, estudiantes de todas las edades suelen encontrar difícil la lectura de los textos científicos, y las principales dificultades que encuentran son lingüísticas, afirman M. A. K. Halliday \& J. R. Martin, en Writing Science Literacy and Discourse Power. London: The Palmer Press, 1993.

Este principio de rehuir la ambigüedad, la polisemia y los juicios de valor, a la par que lograr el rigor, la concisión y la propiedad, implica el uso de formas lingüísticas propias de la exposición, como son:

\section{- Tendencia a la precisión léxica y al rigor.}

- Tecnicismos de la materia de que se trate, cultismos y préstamos lingüísticos. Por tal motivo, se evitan las palabras-baúl que son poco precisas, o las de uso cotidiano, como: cosa, tiene, hacer, etc.

- Adjetivación específica, pospuesta y valorativa. Este tipo de adjetivación supone una particularización del sustantivo; es decir, agrega a la significación del sustantivo algo que no está necesaria o naturalmente comprendido en ella. 
Esta característica lingüistica es muy frecuente en los textos que pretenden exponer y explicar información, en tanto que introducen información nueva en el texto o discurso.

- Marcas de modalización o modalizadores. Estos indicadores textuales introducen el punto de vista del emisor en el discurso, bien mediante verbos modales (poder, desear), de opinión (creer, opinar) o performativos (prometer, jurar); bien mediante otros procedimientos, como la cuantificación, la calificación mediante adjetivos o elementos equivalentes, el uso de exclamaciones, o interrogaciones de carácter retórico, los adverbios oracionales, etc. (Ver ÁLVAREZ, 2005). En este tipo de textos abundan los modalizadores asertivos, aquellos que plantean la veracidad o falsedad de un enunciado.

Estas marcas suponen la presencia del enunciador en el enunciado, mediante la "huella" que deja en el enunciado, lo que implica un juego de tensiones entre la objetividad que caracteriza a estos discursos y la subjetividad o valoración que imprime la presencia del enunciador a través de estas marcas de modelización. Conviene tener siempre presente estas marcas de manera que la reflexión ayude a regular la presencia de las mismas en el texto. Veamos algunos ejemplos:

[DCCSS1] Una cultura geográfica para todos: el papel de la geografía en la Educación Primaria y Secundaria

Desde principios de la Edad Moderna, por lo menos, la Geografia ha tenido siempre una clara función educativa, compatible, no obstante, con sus otras finalidades como saber práctico y aplicado al servicio del Estado, pero no menos importante que éstas.

[G1] El comportamiento mensual de los ríos

Conviene insistir (sic) que los precipitados regímenes fluviales corresponden a comportamientos mensuales de ríos que drenan ambientes climáticos muy homogéneos. Obviamente, una cuenca de drenaje, cuando integra sucesivos sectores climáticos, ofrece un régimen complejo o compuesto.

[G2] Modificaciones derivadas de la actividad humana: la lluvia ácida

El hombre a través de sus actividades se ha convertido en agente perturbador del ambiente físico y de manera inconsciente interfiere en la precipitación al menos de dos maneras muy claras: edificando ciudades y por medio de los contaminantes que vierte a la atmósfera.

[G3] Evolución del mapa político europeo

El número de estados europeos podría aumentar, ya que varios de ellos albergan minorías que aspiran a tener mayor grado de autonomía e independencia. 
[G3] Consecuencias de la fragmentación

La terrible guerra intestina yugoslava fue claramente avivada, o incluso desencadenada, por la política exterior de EEUU. El resentimiento había crecido entre eslovenos, croatas, albaneses y musulmanes contra la severa represión de los sentimientos nacionales y la burda perturbación de las economías regionales llevada a cabo sistemáticamente por el régimen controlado por los servios.

[H1] Los cambios de la sociedad campesina y el modo de vida urbano. Los primeros síntomas de la modernización

Entre todos los cambios que se anunciaban, y que alterarian el paisaje y la vida colectiva en la ciudad, destacó_el automóvil y su modelo protagonista, el 600, convertido en todo un símbolo de las mutaciones en las costumbres y los valores.

[H2] Historia atlántica. Un debate historiográfico en Estados Unidos

Y es cierto que la Historia Atlántica está invadiendo todas las parcelas del saber tanto en Europa como en las Américas.

[HA1] La Edad de Bronce

Pocas contribuciones a la historia de la Humanidad han tenido tanta trascendencia en el dominio de la naturaleza como la adquisición de los medios materiales y técnicos para producir objetos de bronce.

[HA1] Stonehenge y la cultura del vaso campaniforme

Se viene entendiendo que la imponente obra arquitectónica en el ancestral y sagrado sitio de Stonehenge pudo ser consecuencia de una desacralización del lugar.

[HA2] Conquistadores del mundo

Se siente uno casi como una hormiga al pasear por Roma entre sus enormes pilares.

[HA2] Sobre el Panteón

Conozco pocos edificios que comuniquen tal impresión de armonía serena. No se experimenta ninguna sensación de pesantez.

[HA3] La arquitectura del siglo XVIII en Potosí

Difícil será encontrar un modelo de campanario comparable a la espadaña de la Compañía, cuya silueta se recorta sobre el limpio cielo de Potosí con la grandiosidad y el empaque de un arco de triunfo de la Roma antigua (fig. 501). 


\subsection{En el nivel morfológico}

- Predominio del presente, con valor de atemporalidad. Suelen abundar también los verbos estativos, así como la cópula ser. Predominan igualmente las formas verbales no personales o impersonales, por tratarse de textos que persiguen la universalización y la cientificidad.

Todos estos fenómenos lingüísticos y textuales constituyen lo que podríamos denominar la "gramática de la exposición de información", de manera que, en la medida en que se conozcan y practiquen dichas características o peculiaridades, se conseguirá que los alumnos interioricen progresivamente tales regularidades y automaticen la escritura de dichos textos, en los aprendizajes y en las enseñanzas de todas las materias del currículo y en todos los niveles del sistema educativo, y fuera del mismo.

\section{CONCLUSIONES}

Primera. Los textos académicos responden a una estructura mental y se caracterizan por una serie de regularidades lingüísticas y textuales que es necesario aprender, y, en consecuencia, hay que enseñarlas, en cada materia del currículo (concepción instrumental de la lengua), con el fin de entender mejor y de producir con mayor competencia textos de especialidad. La reflexión que merecen tales características o peculiaridades, principalmente relacionadas con la función discursiva de explicar y exponer información, merecerá mayor detenimiento en la asignatura de Lengua Castellana y Literatura, por tratarse de un metalenguaje necesario para comprender mejor y expresarse con mayor competencia.

Segunda. Este trabajo no persigue un estudio cuantitativo del corpus manejado; sin embargo, podemos afirmar que los fenómenos lingüísticos y textuales que aparecen con mayor frecuencia en los textos analizados, y que podrían constituir la "gramática de la exposición", son los siguientes: formas supralingüísticas; definiciones, descripciones; citas y referencias a otros estudios; ilustraciones y ejemplificaciones; enumeraciones y comparaciones; vocabulario específico; adjetivación descriptiva y valorativa (en mayor medida, la primera); reformulaciones intradiscursivas; marcas de explicación (aposiciones, dos puntos, rayas, paréntesis, y el que explicativos); deícticos espacio-temporales; conectores lógicos; modalizadores del discurso; y tiempo del discurso expresado en presente con valor de atemporalidad.

Tercera. El conocimiento y la ejercitación de las particularidades de la escritura académica de textos de ciencias sociales, por parte de los estudiantes, favorecen una mayor conciencia de la escritura, ya que se registran en menor medida marcas propias de la oralidad, imprecisiones o vaguedades, redundancias o repeticiones en el uso de términos, entre otros fenómenos. Esto le hace al estudiante más competente en el área de conocimiento en cuestión, tanto en la adquisición del contenido informativo, como en el dominio del género discursivo correspondiente y en la 
exposición de sus producciones, así como en la transformación del conocimiento que lleva consigo la práctica de la escritura reflexiva.

Cuarta. La escritura expositiva o académica propicia el rigor y la concisión necesarios en la producción de textos académicos, además de permitir al estudiante la adquisición de los rasgos predominantes de esta modalidad de escritura. Ello confirma la necesidad de intervenir en las aulas para proporcionar información acerca de las peculiaridades de estos textos y propiciar su ejercitación, de manera que los alumnos tomen conciencia de dichas regularidades y las vayan utilizando progresivamente en la producción de sus textos.

\section{REFERENCIAS BIBLIOGRÁFICAS}

ADAM, J.-M. (1992): Les textes: Types et prototypes, Paris, Nathan.

ÁLVAREZ, T. (2001): Textos expositivo-explicativos y argumentativos, Barcelona, Octaedro.

--- (2005): Didáctica del texto en la formación del profesorado, Madrid, Síntesis.

--- (2010): Competencias básicas en escritura, Barcelona, Octaedro.

BAZERMAN, Ch. et al. (2005): Referente Guide to Writing Across the Curriculum, Indiana, Pador Press.

BEREITER, C. y M. SCARDAMALIA (1987): The Psychology of Written Composition, NJ Erlbaum, Hillsdale.

CAMPS, A. (2003): Secuencias didácticas para aprender a escribir, Barcelona, Graó.

DIDACTEXT, Grupo (2012): Plataforma "RedacText", guía on line para ayudar a redactar (http://www.redactext.es)

GARCÍA PAREJO, I. (coord.) (2011): Escribir textos expositivos en el aula. Fundamentación teórica y secuencias para diferentes niveles, Barcelona, Graó.

SÁNCHEZ MIGUEL, E. (1993): Los textos expositivos, Madrid, Santillana.

TEBEROSKY, A.: "El texto académico". En M. CASTELLÓ (Coord.): Escribir y comunicarse en contextos científicos y académicos. Conocimientos y estrategias, Barcelona, Graó, pp. 17-46.

WRAY, D. y M. LEWIS (1997): Aprender a leer y escribir textos de información, Madrid, Morata, 2000.

\section{Referencias del corpus de textos}

[DCCSS1] "Una cultura geográfica para todos: el papel de la geografía en la Educación Primaria y Secundaria". En A. Moreno Jiménez y Mª J. Marrón Gaite (eds.). Enseñar geografía. De la teoría a la práctica. Madrid: Síntesis, 1995, pp.43- 57.

[DCCSS2] "Los contenidos en la enseñanza de la historia". En J. Prats (Coord.). Didáctica de la Geografía y la Historia. Barcelona: Graó, Vol.II, 2011, pp. 3147. 
[DCCSS3]. "La selección y secuenciación de los contenidos sociales". En Benejam, P. y J. Pagès. Enseñar y aprender ciencias sociales. Geografia e Historia en la Educación Secundaria. ICE Universidad de Barcelona/Horsori, pp. 71-95.

[G1] "Las aguas”. En R. Méndez y F. Molinero. Geografía de España. Barcelona: Ariel, 1993, pp. 208-253.

[G2] "Características de la precipitación". En J. M Cuadrat y M ${ }^{\mathrm{a}}$ F. Pita. Climatología. Madrid: Cátedra, $3^{\mathrm{a}}$ ed., 2004, pp. 160-178.

[G3]. "Evolución del mapa político europeo". En W. H. Berentsen. Europa contemporánea. Un análisis geográfico. Barcelona: Ediciones Omega, 2000, pp. 129-158.

[H1]. "La sociedad española. Pautas tradicionales y síntomas de modernización". En J. Martínez Martín (Coord.). Historia de España. Siglo XX (1939-1996), Madrid: Cátedra, $2^{\mathrm{a}}$ ed., 2003, pp. 96-105

[H2]. "Historia atlántica. Un debate historiográfico en Estados Unidos". En C. de la Guardia.Herrero. Revista Complutense de Historia de América, 2010, Vol. 36.7, pp. 151-159.

[H3]. "La Segunda Guerra Mundial". En A. M. Moral Roncal: Historia Universal Contemporánea II. De la Primera Guerra Mundial a nuestros días. (Javier Paredes (Coord.), Barcelona: Ariel, $7^{\mathrm{a}}$ ed., 2011, pp. 714-735.

[HA1] "La Edad de Bronce". En Fernández, $\mathrm{M}^{\mathrm{a}}$ del C.: La Edad de los Metales. Madrid: Grupo 16, Col. Historia del Arte, no 4, pp. 8-47.

[HA2] "Conquistadores del mundo". En Gombrich. E. Historia del Arte. Madrid: Alianza Forma, 1979, pp.93-103.

[HA3] "La arquitectura del siglo XVIII en Potosí". En Diego Angulo Íñiguez. Historia del arte hispanoamericano. Barcelona: Salvat Editores, 1956, pp. 503534. 